\title{
Versöhnung von Nation und Demokratie? Oder: Ist die europäische Erfahrung universalisierbar?
}

von Andreas Wirsching

Zwar kann die westliche Demokratie wohl immer weniger selbstverständlich als Modell für künftige Entwicklungen im Weltmaßstab dienen, doch stellt sich gleichwohl die Frage, ob die europäische Antwort auf die Spannungen zwischen den Konzepten „Nation“ und „Demokratie“ als universalisierbar gelten darf. Der nachfolgende Beitrag widmet sich den unterschiedlichen Wahrnehmungen des europäischen „Erfolgsmodells“, diskutiert seine tiefe Verankerung in den (häufig divergenten und ungleichzeitigen) nationalstaatlichen Erfahrungen und verweist auf die Konfliktpotentiale zwischen den Wilson'schen Prinzipien der demokratischen Regierungsform und des Selbstbestimmungsrechts der Völker. Obwohl eine Vorbildfunktion der europäischen Entwicklungsgeschichte angesichts der katastrophalen Erfahrungen des Kontinents eher verneint werden muss, könnte die spezifische Antwort, die Europa auf die Erfordernis eines postnationalstaatlichen Ordnungsmodells gefunden hat, durchaus auch im globalen Maßstab eine Wirkung entfalten.

While Western democracy is increasingly losing its position as a reference model for future political developments on a world-wide scale, the question remains whether Europe's answer to the tensions between the concept of "nation" and "democracy" namely economic and political integration - can be seen as universally applicable. This contribution seeks to analyse different perspectives on the European "model of success", discusses its deep linkages with the experience of nation-state development and sheds light on potential conflicts between Wilson's principles of (territorial) democracy and (national) self-determination. Even though Europe's overall development can hardly be cited as a general model due to the continent's catastrophic historical experiences, her specific answer to the need for a system of post-national order may yet prove to be of global relevance.

I.

Manches spricht dafür, den Umbruch von 1989/90 als Teil einer „dritten“, globalen Welle der Demokratie zu interpretieren, die neben Europa auch Lateinameri- 
ka, Asien und (Süd-)Afrika erfasste. ${ }^{1}$ Nach dem Ende der autoritären Regime in Griechenland, Portugal und Spanien während der 1970er Jahre brachte sie mit dem Zusammenbruch des Kommunismus und unter dem Jubel der Massen in ganz Europa die Demokratie zur Geltung. Die damalige Euphorie ist inzwischen allerdings verflogen; global betrachtet scheint die Demokratie eher wieder auf dem Rückzug zu sein. Und angesichts überall zu beobachtender Tendenzen zu Überkomplexität, Politikverdrossenheit und Populismus mehren sich die Stimmen, die zugleich auch eine schleichende innere „Erosion“ der Demokratie konstatieren. Sie sprechen von „Postdemokratie“ und „Transformation“ und empfehlen zumindest eine „Modernisierung“ der Demokratie. ${ }^{2}$

Vor diesem Hintergrund erscheint es immer weniger selbstverständlich, dass die westliche Demokratie als Modell für künftige Entwicklungen im Weltmaßstab dienen kann. Der Aufbruchsstimmung von 1990 folgt eine Phase der Unsicherheit und der tastenden Selbstvergewisserung. Schon fragt auch die große Publikumspresse, ob sich nicht alternative politische Systeme möglicherweise als langfristig leistungsfähiger herausstellen könnten als die westliche Demokratie: „In Singapur oder China scheinen sie auf je eigene Weise zu gewährleisten, was immer mehr demokratische Politiker vermissen lassen: professionelles politisches Management und strategische Planung, die sich nicht an schwankenden Stimmungen orientiert. “"

Abgesehen davon, dass solche Aussagen in einem merkwürdigen Kontrast stehen zur fast gleichzeitig erfolgten Verleihung des Friedensnobelpreises an einen inhaftierten chinesischen Vorkämpfer für Demokratie und Menschenrechte, wirft der gegenwärtige innerdemokratische Krisendiskurs doch auch sehr grundsätzliche Fragen auf. Angesichts einer internationalen politikwissenschaftlichen Dauerdebatte, in der sich demokratietheoretische, empirisch-analytische und normative Elemente überlagern, ist es daher sinnvoll, sich einmal mehr der historischen

1 Huntington, S.: The Third Wave. Democratization in the Late Twentieth Century, Norman (Oklahoma), 1991. Zum Umbruch von 1989/90 zusammenfassend: Dalos, G.: Der Vorhang geht auf. Das Ende der Diktaturen in Osteuropa, München, 2009.

2 Crouch, C.: Postdemokratie, Frankfurt/M., 2008 (zuerst 2002); Warren, M. E.: A second Transformation of Democracy?, in: Cain B.E. u.a. (Hg.), Democracy Transformed? Expanding Political Opportunities in Advanced Industrial Democracies, Oxford, 2003, 223-249; zusammenfassend Schmidt, M. G.: Demokratietheorien. Eine Einführung, Wiesbaden, 2010, v.a. 495-505. Grundsätzlich anregend: Greven, M.: Die politische Gesellschaft. Kontingenz und Dezision als Probleme des Regierens und der Demokratie, 2. Aufl., Wiesbaden, 2009; siehe ders.: Zukunft oder Erosion der Demokratie?, in: Kaspar H. u.a. (Hg.), Politik - Wissenschaft - Medien (Fs. J.W. Falter), Wiesbaden, 2009, 411-428.

3 Buchsteiner, J.: Demokraten und Meritokraten, in: Frankfurter Allgemeine Zeitung, 13. Oktober 2010. 
Grundlagen des Themas zu vergewissern. Von hier aus lässt sich dann nachhaltig fragen, in welchem historischen Verhältnis die spezifische europäische Erfahrung von Nation und Demokratie mit den aktuellen Welttendenzen stehen könnten.

Trotz mancher alter Probleme und vieler neuer Unsicherheiten in der politischen Gegenwart Europas lässt sich kaum leugnen, dass der Kontinent seit 1990 in seine historisch wohl „glücklichste“ Zeit eingetreten ist: Frieden auf der Basis von Freiheit, Wohlstand kombiniert mit einem erheblichen $\mathrm{Ma}$ an sozialer Sicherheit, kultureller Reichtum gespeist aus Liberalität. Ist das nicht im Grunde eine Art Paradies, in dem, oder zumindest an dessen Schwelle, sich nach wie vor die Mehrheit der Europäer befindet - allen unleugbaren und gewaltigen Ungleichheiten zum Trotz? Tatsächlich fragt man sich: Wer wollte nicht so leben wie die Europäer? Und so betrachtet, kann es eigentlich kaum Einwendungen gegen die Idee geben, Europa stelle ein universalisierbares Entwicklungsmodell dar: gerüstet für globale Herausforderungen und attraktiv als Leuchtturm in einer immer näher zusammenrückenden „Weltgesellschaft“. Etwa so, wie es ein britisches Memorandum aus dem Foreign Office bereits 1926 für das eigene Land formulierte: „We have got all what we want-perhaps more. Our sole object is to keep what we have and live in peace. "4

Aber so einfach ist es selbstverständlich nicht, und dies liegt keineswegs nur an Oberflächlichkeiten wie etwa der Tatsache, dass dem europäischen Modell selbst genügend politisch-kulturelle Probleme anhaften oder dass es im Zeitalter der Globalisierung um seine ökonomisch-materielle Basis bangen muss. Aus historischer Sicht ist es ohnehin problematisch, von einem „Entwicklungsmodell“ Europa zu sprechen. Allzu sehr birgt dies zum einen die Gefahr einer essentialistischen Konstruktion, die gleichsam nur von innen heraus erfolgt und den Blick von außen ignoriert. Zum zweiten droht eine teleologische Sichtweise, die den heutigen Zustand Europas als Ziel der Geschichte betrachtet: als ein Ziel, auf das die historischen Entwicklungspotentiale und Entwicklungsprozesse zwingend hingearbeitet haben. Es gilt daher davor zu warnen, die Geschichte Europas in diesem Sinne gleichsam als eine Whig interpretation of history zu lesen. Denn damit erlägen wir der Versuchung, die Vergangenheit unserer heutigen Sicht unterzuordnen und an der Elle der Gegenwart zu messen. Das Ergebnis wäre

4 Memorandum on the Foreign Policy of His Majesty's Government, with a List of British Commitments in their Relative Order of Importance, in: Documents on British Foreign Policy, Ser. Ia, Bd. 1: The Aftermath of Locarno 1925-1926, London, 1966, 846-881, hier 846. 
eine eher flache europäische Fortschrittsgeschichte, teleologisch reduziert und ihrer Komplexität entkleidet. Eine solche Geschichte wird zum eurozentrischen Mythos, zur bloßen Meistererzählung. Kein Wunder daher, dass die geschichtswissenschaftliche Kritik an entsprechenden Tendenzen inzwischen sehr deutlich vernehmbar ist. ${ }^{5}$

Die zugrunde liegende Schwierigkeit wird deutlich, wenn man die für Europa gültigen „Erfolgsfaktoren“ diskutiert und im selben Zusammenhang die Frage stellt, welche Bedeutung sie für andere Kulturkreise haben können. Denn was können solche für Europa gültigen Erfolgsfaktoren sein?

Jenseits aller anderen denkbaren Möglichkeiten sind sie gewiss zeit- und standortabhängig. Im Jahre 1900 etwa hätte kaum ein europäischer Intellektueller, Unternehmer oder Politiker gezögert, die Segnungen der europäischen Wissenschaft und Technik, Zivilisations- und Lebensformen als unverbrüchlich gültige „Erfolgsfaktoren“ zu deklarieren. Zugleich aber würde er sie wohl imperialistisch gewendet und als naturnotwendige Basis europäischer Weltüberlegenheit gewertet haben. Auch hätte so mancher Intellektueller nicht gezögert, die neuesten Erkenntnisse der Sozialeugenik und Rassenforschung als ,gültige Erfolgsfaktoren“ des europäischen Entwicklungsmodells zu betrachten.

Tatsächlich ist ja die Rede von Europa oder dem europäischen „Modell“ in weiten Teilen der Welt mit der Erfahrung des europäischen Kolonialismus und Imperialismus konnotiert - oder sogar kontaminiert. ${ }^{6}$ Seine modernen Ingredienzien - Nation und Demokratie - haben eine dezidiert exklusive Wirkung entfaltet und die Kolonialvölker aus der sich bildenden Gemeinschaft kategorisch ausgeschlossen. Schon die französische Nationalversammlung verbannte 1789 die farbige Sklavenbevölkerung in Santo Domingo aus dem zugleich doch so emphatisch zelebrierten Nationsbegriff. Die natürlichen Menschenrechte, die sich zunächst innerfranzösisch, sodann innereuropäisch zu einer so scharfen emanzipativen Waffe schmieden ließen, galten nicht für die Sklaven - und zunächst auch nicht für Frauen.

5 Zur Kritik vgl. exemplarisch: Osterhammel, J.: Europamodelle und imperiale Kontexte, in: Journal of Modern European History 2 (2004), 157-182; Sachsenmeier, D.: Recent Trends in European History: The World beyond Europe and Alternative Historical Spaces, in: Journal of Modern European History 7 (2009), 5-25. Als großer Versuch, europäische Geschichte als Teil einer neuen Form von Weltgeschichte zu betrachten, Osterhammel, J.: Die Verwandlung der Welt. Eine Geschichte des 19. Jahrhunderts, München, 2009.

6 Exemplarisch und einflussreich: Chakrabarty, D.: Provincializing Europe: Postcolonial Thought and Historical Difference, Princeton (N.J.), 2000. 
Im postkolonialen Zeitalter kann Europa daher nur dann als relevantes Entwicklungsmodell Geltung beanspruchen, wenn es selbst-reflexiv ist, das heißt, wenn sich seine Vertreter und Stichwortgeber seiner historischen Brüche und Schattenseiten, aber auch seiner Ambivalenzen und zwiespältigen Folgen bewusst sind.

Im folgenden werden daher einige kritische Überlegungen zur historischen Dimension des Entwicklungsmodells Europa angestellt. Die Konzentration auf die Leitbegriffe Nation - Demokratie - Integration rückt jene drei Motive in den Mittelpunkt, die heute am ehesten als die langfristig wirksamen Elemente eines politischen Entwicklungsmodells gelten können. Tastsächlich gilt es zu betonen, dass dies die heutige Sicht ist. Und niemand kann sicher sein, ob nicht morgen vielleicht andere Elemente Priorität einfordern - in Frage kämen etwa Konzepte wie Föderation und Multiethnizität, Genossenschaft und Volksversammlung.

\section{II.}

Die Nation ist bekanntlich eine ,vorgestellte Gemeinschaft“ - so die mittlerweile klassische, kulturwissenschaftlich allgemein akzeptierte Formulierung von $\mathrm{Be}$ nedict Anderson. Zugleich freilich bedarf diese Vorstellung, wenn sie denn konkret sein will, auch einer konkreten Begrenzung. ${ }^{7}$ Dem entspricht Niklas Luhmanns Feststellung, wonach jede territoriale Grenzsetzung im Bewusstsein weiterer, dahinterliegender Grenzen erfolgt: „Denn auf der anderen Seite jeder Grenze gibt es wiederum Länder mit Grenzen, die ihrerseits eine andere Seite haben. “8 Das bedeutet: Nationen brauchen territoriale Grenzen und suchen sich damit ihre staatliche Form. Nationen in ihrem staatlichen Gehäuse, Nationalstaaten also, sind ein grundlegendes Prinzip, nach welchem sich die neuere und neueste Geschichte Europas gestaltet.

Freilich müssen wir, wenn wir nach den Wurzeln der europäischen politischen Kultur fahnden, noch weiter zurückgehen und nach der Entstehungsgeschichte des modernen Staates selbst fragen. Tatsächlich ist der moderne Staat eine gleichsam europäische Erfindung, die sich über die Welt verbreitet hat. Seine Wurzeln liegen letztlich in der jüdisch-christlichen Vorstellung der Transzendenz. Denn erst die radikale Idee des Jenseitigen erlaubt die säkulare Organisati-

7 Anderson, B.: Die Erfindung der Nation. Zur Karriere eines folgenreichen Konzepts, Frankfurt/M./New York, 1996 (zuerst englisch 1983), 16.

8 Luhmann, N.: Die Gesellschaft der Gesellschaft Bd. 1, Frankfurt/M., 1997, 150. 
on des Diesseitigen. ${ }^{9}$ Gottkönige und sakrale Universalreiche haben im europäischen Entwicklungsmodell keine Chance. Stattdessen differenziert sich der Staat als innerweltliches Subsystem aus, das daher auch zwingend einer stetigen rechtlichen Regelung unterliegt.

Die Vor- und Frühformen moderner Staatlichkeit bestanden in Europa also bereits, als die Nation als neuer historischer Akteur die Bühne betrat. Aber die Entwicklungsbedingungen der europäischen Nationalstaaten waren, wie schon Theodor Schieder aufgezeigt hat, ${ }^{10}$ sehr unterschiedlich. Im west- und auch nordeuropäischen Raum entfalteten sich die Nationen schon im 18. Jahrhundert und konstruierten ihre Identität in einem bereits monarchisch weit entwickelten und institutionalisierten Staat. Dem entgegen steht der mittel- und südeuropäische Raum. Hier bildeten sich die Nationen zunächst innerhalb eines historischen Gefüges von Einzelstaaten. Eine Vielzahl von Grenzen durchschnitt jene Räume, welche die Nationen für sich reklamierten. Die Begründung eines Nationalstaats erforderte daher die - gegebenenfalls gewaltsame - Zusammenfügung bereits bestehender staatlicher Räume.

Idealtypisch stehen sich also der westeuropäische Raum mit England und Frankreich einerseits und der mittel- und südeuropäische Raum mit Deutschland und Italien andererseits gegenüber, um nur die wichtigsten Fälle zu nennen. Im dritten hier zu nennenden Raum, im östlichen Mitteleuropa sowie in Südosteuropa, liegen die Dinge wiederum anders: Hier korrespondiert die Nationsbildung mit einem Prozess der staatlichen Sezession. Das heißt, auch die mittel-, ost- und südosteuropäischen Nationen mussten sich ihren Staat erst erkämpfen, nicht aber wie in Deutschland und Italien durch Zusammenfügung älterer monarchischer oder städtisch-republikanisch gewachsener Staatsgebilde, sondern durch die Abspaltung von einem der drei großen östlichen Vielvölkerreiche - Habsburgermonarchie, Osmanisches Reich und Russland. Die sezessionistischen Staatsgründungen der sowjetischen Nachfolgestaaten Anfang der 1990er Jahre knüpften noch einmal an diese Tradition an. ${ }^{11}$

9 Vgl. Reinhard, W.: Was ist europäische politische Kultur? Versuch zur Begründung einer politischen Historischen Anthropologie, in: Geschichte und Gesellschaft 27 (2002), 593-616, hier v.a. 601.

10 Schieder, Th.: Der Nationalstaat in Europa als historisches Phänomen (1964) sowie Typologie und Erscheinungsformen des Nationalstaats in Europa (1966), beides in: ders.: Nationalismus und Nationalstaat. Studien zum nationalen Problem im modernen Europa, hg. v. Otto Dann und Hans-Ulrich Wehler, Göttingen, 1991, 65-86 u. 87-101, hier v.a. 68-72.

11 Zur Dekomposition der Sowjetunion vgl. Huber, M.: Moskau, 11. März 1985. Die Auflösung des sowjetischen Imperiums, München, 2002; Zubok, V. M.: A Failed Empire. The Soviet Union in the Cold 
Zwischen dem west- und nordeuropäischen Raum einerseits und den anderen Räumen der europäischen Geschichte andererseits bestanden also fundamentale Unterschiede. Anders als in England, Frankreich oder Skandinavien vollzog sich die Nationsbildung in Deutschland, Italien und im ostmitteleuropäischen Gebiet nicht im Gehäuse eines gefestigten Staates. Vielmehr waren es die Nationen, die ihren Staat erst suchen, seine Grenzen erst definieren mussten. Diese Nichtidentität von Nation und Staat prägte die europäische Geschichte im 19. und in der ersten Hälfte des 20. Jahrhunderts. Sie barg den Keim schwerster Konflikte und trug im Zeitalter der Weltkriege maßgeblich zur Entfesselung der Gewalt bei. Erinnert sei etwa an den türkisch-griechischen Krieg von 1919 bis 1923 und den am Ende im Frieden von Lausanne gewählten Bevölkerungsaustausch: eine „Lösung“, die keine Lösung war, sondern faktisch die Deportation von mehr als anderthalb Millionen Menschen bewirkte.

Erinnert sei ferner an die Spannungen, die das Versailler System im östlichen Mitteleuropa hervorrief: Spannungen, die sich daraus ergaben, dass die Pariser Vorortverträge eben keine National-, sondern Nationalitätenstaaten schufen. Diese auf den Ruinen der kaiserlichen Großreiche errichteten Staaten blieben Vielvölkergebilde ebenso wie die früheren ,Völkergefängnisse“, das heißt eben jene Kaiserreiche, die sie ersetzten. Tatsächlich gab es im östlichen Mitteleuropa nach 1918 keine Nation, von der nicht Teile außerhalb ihrer Staatsgrenzen, und keinen Staat, in dem nicht mehrere nationale Minderheiten lebten.

Und schließlich sei erinnert an die verbrecherischen Umsiedlungs- und Vertreibungspläne, mit denen das nationalsozialistische Deutschland im Zweiten Weltkrieg die Grenzen einer germanischen Nation weit nach Osten verschieben und damit den Lauf der europäischen Geschichte in einer rassistischen Utopie stillegen wollte. $^{12}$

Alles dies gehört zur Geschichte der Nation und des Nationalen in Europa. Dort wo in Europa ein ethnisch verengtes Verständnis von Nation vorherrschte, entschwand auch das Versprechen der Freiheit. Und allzu häufig erhob hinter dem Begriff der Nation menschenverachtende Gewalt ihr Haupt. Im JugoslawienKonflikt der 1990er Jahre ist Europa von diesem Gespenst der Vergangenheit noch einmal eingeholt worden. Kein Zweifel, europäische Nationsbildung und

War from Stalin to Gorbachev, Chapel Hill (N.C.), 2007, v.a. 303-335; Brown, A.: Aufstieg und Fall des Kommunismus, Berlin, 2009, v.a. 731-763.

12 Pars pro toto: Aly, G.: Endlösung: Völkerverschiebung und der Mord an den europäischen Juden, 3. Aufl., Frankfurt/M., 2005. 
europäischer Nationalstaat sind - historisch betrachtet - höchst problematische Angelegenheiten. Aber die Sache wird noch weitaus komplexer, wenn wir uns dem zweiten Stichwort zuwenden, der Demokratie.

\section{III.}

Zwar wird kaum jemand bezweifeln, dass die Demokratie die wohl wichtigste politische Errungenschaft des europäischen Entwicklungsmodells darstellt. Zugleich aber ist die Geschichte der europäischen Demokratie untrennbar mit der Geschichte von Nation und Nationalstaat verflochten - und das macht es noch schwieriger, das politische Modell Europa mit einfachen Kategorien zu bewerten. So wie die Nation ist auch die Demokratie nur in einem abgrenzbaren Raum vorstellbar. Die Ausübung von Freiheit setzt die abgrenzbare politische Gemeinschaft voraus. ${ }^{13} \mathrm{Ja}$ mehr noch: Die europäische Geschichte lehrt, dass die kulturelle Verankerung der Nation in einem vorgestellten und unumstrittenen, das heißt klar umgrenzten Territorium eine wichtige Voraussetzung der Demokratie darstellt.

Dem entspricht es, wenn die Demokratien des Westens im 19. und frühen 20. Jahrhundert dort am stabilsten waren, wo die Identität von Nation und Staat am weitesten gediehen war. Dagegen verfing sich der von den Alliierten im Ersten Weltkrieg auf die Fahnen geschriebene große Anlauf zur Demokratisierung im neugewebten Netz der territorialen und ethnischen Grenzen. Zwar trieben der Erste Weltkrieg und die staatliche Sezession die Nationsbildung im östlichen Mitteleuropa katalytisch, romantisch und gewaltsam voran. Auf den ersten Blick schien hier also ein großer konstruktiver Akt neue Identitäten von Nationen und Staaten hergestellt zu haben, die zugleich reif für die Demokratie waren, etwa im Sinne einer „Republikanisierung“ à la française. Und in den Pariser Vorortverträgen insistierten die Verbündeten auf der Anwendung des westlichen Modells: Ausdehnung des Wahlrechts, Demokratisierung der Parlamente und das Bürgerrecht für Minderheiten. ${ }^{14}$

Obgleich mit großen Hoffnungen begrüßt, endete der demokratische Aufbruch von 1918 schon bald in nationaler Frustration und gesteigertem Hass. Allzu häufig bildete der Irredentismus den größten gemeinsamen Nenner der führenden

13 Vgl. Di Fabio, U.: Der Verfassungsstaat in der Weltgesellschaft, Tübingen, 2001, 32.

14 Janos, A. C.: East Central Europe in the Modern World. The Politics of the Borderlands from Pre- to Postcommunism, Stanford (Calif.), 2000, $100 \mathrm{ff}$. 
Schichten. ${ }^{15} \mathrm{Zu}$ keinem Zeitpunkt erreichte das östliche Mitteleuropa während der Zwischenkriegszeit eine hinreichende Stabilität, die doch die notwendige Voraussetzung für das Reifen der Demokratie gewesen wäre. Es gab dort keine Demokratie, die sich nicht zugleich mit territorialen und ethnischen Problemen auseinanderzusetzen hatte.

Die Demokratie ist also im europäischen Entwicklungsmodell nicht einfach als lineare Fortschrittsgeschichte enthalten. Der amerikanische Soziologe Michael Mann hat geradezu von der „dunklen Seite der Demokratie“ gesprochen und damit in erster Linie auf die europäische Entwicklung gezielt. ${ }^{16}$ Diese dunkle Seite drängte sich dann in den Vordergrund, wenn unklar blieb, wer eigentlich das „Volk“ war, das sich zum Souverän aufschwang. War es der Ethnos, der sich sprachlich und kulturell einheitlich begriff? Oder war es der Demos, der unterschiedliche gesellschaftliche Interessen in sich vereinigte und sie organisierte? Wenn beide Konzepte der „Demokratie“ sich vermischten, so Manns These, dann war Gefahr im Verzug: so etwa im Europa der Zwischenkriegszeit, für das Präsident Wilson einerseits die repräsentative Demokratie ausrief, andererseits aber die nationale Selbstbestimmung einforderte - zum damaligen Zeitpunkt ein in sich widersprüchliches Konzept. Die Völker Europas konnten sich nicht entscheiden, was Vorrang haben sollte: die pluralistische Repräsentation des Demos unter Einschluss nationaler Minderheiten oder die ethnische Homogenisierung des Volkes unter Ausschluss anderer Nationalitäten. Während das westlich geprägte Europa des Versailler Systems und des Völkerbundes an der Fiktion des demokratisch-pluralistischen Nationalstaats festhielt, gewann in Mittel- und Osteuropa das Konzept der ethnischen Homogenität im Zeitablauf zunehmend die Oberhand. Und wenn Staatsbürgerschaft zunehmend ethnisch definiert wurde, dann verengten sich auch die Möglichkeiten einer internationalen Friedensordnung.

Verheerend waren im Grunde schon die Zahlen der Zwischenkriegszeit: Bis 1926 gab es bereits ein veritables Problem von Flüchtlingen oder zumindest Entwurzelten. Hierzu gehörten die 1,5 Millionen Griechen und Türken, die 1923 aufgrund des Friedens von Lausanne zwischen beiden Nationalstaaten ausgetauscht wurden; 280.000 Personen wechselten zwischen Griechenland und Bulgarien; und die Zahl der Polen, Ukrainer, Russen, Ungarn, Balten und Deutschen, die ihre Heimat verloren, belief sich insgesamt auf weit mehr als fünf

15 Vgl. Molnar, M.: Histoire de la Hongrie, 2. Aufl., Paris, 1996, 345.

16 Mann, M.: The Dark Side of Democracy. Explaining Ethnic Cleansing, Cambridge, 2005. 
Millionen. In den Augen des Generalsekretärs des Völkerbundes, Carlile Macartney, gab es tatsächlich nur wenige Auswege aus dem Dilemma: Entweder die Revision der Grenzen oder den Austausch bzw. die forcierte Emigration der Bevölkerung. „The experience of all attempts to solve the minority problem by getting rid of the minority have thus proved thoroughly discouraging", schrieb Macartney 1934. "Frontier revision is applicable only in certain cases. Exchange has achieved dubious results at the cost of great sufferings. Assimilation has failed altogether, since the minorities have not been assimilated". ${ }^{17}$

Die Demokratie entwickelte sich also in Europa keineswegs einlinig und unproblematisch, sondern in ganz unterschiedliche Richtungen. Im nordwestlichen Teil des Kontinents, wo die Identität von Nation, Staat und Territorium bereits langfristig gesichert war, überwog auch nach 1918 eine pluralistische Auffassung von Staat und Gesellschaft. In Mittel- und Osteuropa, wo die territorialen Grenzen traditionell fließend und eine Identität von Staat und Nation noch nicht erreicht war, dominierte dagegen die Idee des Ethnos. Der Begriff der Demokratie konnte in diesem Zusammenhang mit ganz anderen Inhalten gefüllt werden, als wir sie heute akzeptieren würden. Verwiesen sei nur auf Carl Schmitts in Deutschland populäre Ansichten von einer plebiszitär-charismatisch begründeten Führerdemokratie. Aber auch die autoritären Überformungen der Nachkriegsdemokratien in Ländern wie Polen oder Ungarn wären hier zu nennen.

Historisch betrachtet sind also im europäischen Entwicklungsmodell Nation und Demokratie in durchaus prekärer und uneindeutiger Weise miteinander verflochten. Offenkundig braucht die Demokratie eine klare Vorstellung von Grenze und Abgrenzung, von Identität und Gemeinschaft und bleibt damit auf die Nation angewiesen. Wenn daher der demokratische Nationalstaat zwar als Ziel gelten kann, so ist der Weg zu ihm gleichwohl gefährlich, weil er an vielerlei Abgründen vorbeiführt.

Entscheidend hierfür ist, dass der Entwicklungsprozess des demokratischen Nationalstaats zwei im Kern unvereinbare Positionen miteinander verbinden muss: Einerseits ist die Nation etwas Individuell-Partikulares. Die Bezugnahme auf die Nation suggeriert zwar die Gleichheit nach innen, aber sie tendiert, wie wir gesehen haben, zum Ausschluss der Nichtdazugehörigen. Andererseits basiert die Demokratie auf allgemeinen Prämissen, die im Kern universalisierbar sind und geradezu zur Universalisierung drängen. Hier nationale und ethnische Zugehö- 
rigkeit, dort allgemeine Menschenrechte und politische Gleichheit: Dies sind im politischen Entwicklungsmodell Europas gleichsam unterschiedliche Systemebenen, die miteinander vermittelt werden wollen. Und die hieraus resultierende Spannung zwischen partikularen und universalen Elementen ist der neueren europäischen Geschichte in ihrer Tiefe eingeschrieben.

Es ist daher kein Zufall, dass in Europa jene Demokratien über das solideste politisch-kulturelle Fundament zu verfügen scheinen, in denen sich die individuelle, national geprägte Identität am nachhaltigsten mit einer universalen „Idee“ verbindet. Eine solche Verschränkung zwischen dem Partikularen und dem Universalen finden wir - einmal mehr - am ehesten in der westeuropäischen Zone. So lässt sich im Falle Großbritanniens von einer langetablierten parlamentarischen Identität sprechen. Sie lag an der Wurzel einer spezifischen Form des Verfassungspatriotismus, der die konstitutionelle Freiheit Englands hervorhob und dessen Anfänge in das 17. Jahrhundert zurückreichen. Liberty, constitution, patriotism bilden hier ein langfristiges Deutungsmuster, das auch nach 1918 seine Orientierungsfunktion nicht einbüßte.

In vergleichbarer Weise ruhte die republikanische Identität Frankreichs auf dem Mythos der Revolution und auf ihrem naturrechtlich begründeten, aber national geformten Sendungsbewusstsein. In beiden Fällen, dem britischen und dem französischen - man könnte den amerikanischen noch hinzunehmen - wurde die nationale Geschichte mit verallgemeinerbaren Werten kombiniert und zu einer überaus deutungsmächtigen Synthese geschmiedet. Dadurch haben die modernen westlichen Demokratien eine entscheidende Vermittlungsleistung erbracht: das geschichtlich Gewordene, das Partikulare, zu akzeptieren und zugleich das Normativ-Universale zu institutionalisieren.

Demgegenüber ruhten weder die deutsche noch die italienische noch die ostmitteleuropäischen Verfassungskulturen auf einer bereits langerprobten Nationalgeschichte, die wie in England, Frankreich und den USA als Fortschrittsgeschichte hätte ideologisiert werden können. Umso problematischer gestaltete sich eben jene Verbindung des National-Partikularen mit dem Demokratisch-Universalen in diesen Räumen Europas. Zu Recht hat etwa Helmuth Plessner das Deutsche Kaiserreich als eine „Großmacht ohne Staatsidee“ bezeichnet. ${ }^{18}$ Dies drückte sich zum Beispiel in den sogenannten „Ideen von 1914“ aus, die zwar den Triumph des Nationalistisch-Partikularen über die universal wirksamen Ideen von

18 Plessner, H.: Die verspätete Nation. Über die politische Verführbarkeit bürgerlichen Geistes, Stuttgart, 1959. 
1789 zelebrieren sollten und doch sehr enggeführt und geradezu muffig wirkten. Eine demokratische Identität, welche die Brücke geschlagen hätte zwischen nationaler Partikularität und universaler Geltung, ist in Deutschland vor 1949 jedenfalls kaum zu entdecken.

Aber auch für das gegenwärtige Europa bildet die Aufgabe, Nation und Demokratie miteinander zu vermitteln, eine der größten Herausforderungen. Denn insbesondere die ostmitteleuropäischen Staaten müssen ja seit dem Aufbrechen des kommunistischen Panzers ihre in den 1920er und 1930er Jahren abgebrochene Identitätsfindung als demokratische Nationalstaaten erst einmal wiedergewinnen und fortentwickeln. Schon vor 1989 bildete die Nation ein entscheidendes Vehikel für die Bewahrung einer eigenen Identität gegenüber dem Sowjetkommunismus. Beispiele hierfür sind insbesondere Polen, Rumänien oder Ungarn. Nach 1989 verknüpften die ostmitteleuropäischen Völker die Abwicklung ihrer kommunistischen Regime mit einer ungeahnten Renaissance des Nationalen. Ihre Demokratien begründeten sie im Gehäuse des Nationalstaats. Nationale Mythen und Symbole, Hymnen und Feiertage, Geschichtserzählungen und Geschichtsdeutungen blühten im Zuge der Befreiung vom Kommunismus auf. Fast überall im östlichen Mitteleuropa fand daher der Rückgriff auf die Nation im Bewusstsein statt, mit ihr den politischen und kulturellen Fortschritt zu sichern. $\mathrm{Zu}$ einem Zeitpunkt, da in Westeuropa die Überwindung nationalstaatlich enggeführter Politik auf der Tagesordnung stand, gewann in Ost- und Südosteuropa die Nation den Status eines Fortschrittskonzepts. Was sich also in den westeuropäischen Staaten im 19. und 20. Jahrhundert in einem langfristigen Prozess vollzog, nämlich die Vermittlungsleistung von Nation und Demokratie, muss in Ostmitteleuropa eminent beschleunigt, in einer Art Zeitraffer, erfolgen.

\section{IV.}

Das europäische Entwicklungsmodell hat auf die Fragen, welche die Spannung zwischen Nation und Demokratie aufwarf, eine historisch einzigartige und bis dato, wie es scheint, erfolgreiche Antwort gefunden - die Integration. Geboren war die Antwort der Integration einerseits aus der bitteren historischen Erfahrung eines sich selbst zerstörenden Kontinents. Zugleich speiste sie sich andererseits aus der Hoffnung und der festen Erwartung, die Substanz der europäischen Geschichte doch retten und ihr eine neue Zukunft geben zu können. Überdies war es die durchaus nüchterne Abwägung nationaler Interessen, die 1950/51 den supranationalen „Quantensprung“ der europäischen Integration ermöglichten - zuerst in Washington und Paris, dann in Bonn sowie schließlich in den anderen Metro- 
polen der sechs Gründungsmitglieder. Tatsächlich vollzog sich die Geschichte der europäischen Integration keineswegs im Gegensatz zum Nationalstaat. Zudem wäre es ahistorisch, allzu schnelle Parallelen etwa zwischen den Strukturen des Heiligen Römischen Reichs und der heutigen Europäischen Union zu ziehen. ${ }^{19}$ Es führt kein direkter Weg vom vor-nationalen Europa der Frühen Neuzeit zur supranationalen europäischen Integration. Im Gegenteil, erst der voll entwickelte, souveräne Nationalstaat war die historische Voraussetzung für die Geschehnisse nach 1945. Die europäische Integration resultierte aus dem Nationalstaat, aber sie resultierte auch aus seiner Hybris. Tatsächlich scheint die europäische Integration nichts anderes zu sein als eine Methode, um unter den Bedingungen der Nachkriegszeit die Existenz des europäischen Nationalstaats zu sichern. ${ }^{20}$

Konkret hieß dies: Die Integration bildete ein Instrument, um den Nationen Europas ein gleichberechtigtes und friedliches Zusammenleben zu ermöglichen, das auf universalisierbaren und daher gleichermaßen anerkannten Prinzipien ruhte. Die Gemeinschaft bildete sich und sie bekannte sich „zu den Grundsätzen der Freiheit, der Demokratie und der Achtung der Menschenrechte und Grundfreiheiten und der Rechtsstaatlichkeit“; sie stärkte ,die Solidarität zwischen ihren Völkern unter Achtung ihrer Geschichte, ihrer Kultur und ihrer Traditionen" und förderte deren „wirtschaftlichen und sozialen Fortschritt“. So steht es in der Präambel des Vertrags von Maastricht.

Tatsächlich scheint es so, als ob das Prinzip der europäischen Integration jenen Ausgleich von partikular-nationalen und universalen Elementen leisten könnte, der für die staatliche Entwicklung der Moderne so bedeutsam ist. Die EU bietet einen koordinierenden Rahmen für demokratische, ethnisch überwiegend homogen gewordene Nationalstaaten. Allerdings ist die Integration in ihrer traditionellen Form, als spezifisch westeuropäisches Nachkriegsprojekt, beendet. Seine Zeit ist abgelaufen in einer Epoche, in der sich die Prioritäten neu gestalten. Französische Europapolitik etwa kann, wenn sie zeitgemäß sein will, nicht mehr primär Deutschlandpolitik sein; vielmehr muss Frankreich in dem neuen, größeren Europa seine Rolle finden. Gleiches gilt für das wiedervereinigte Deutschland, das nicht mehr am östlichen Rand des integrierten Europa liegt, sondern in dessen

19 Eine historische Etappenfolge Europas vom „Alten Reich“ zur Europäischen Union wird gelegentlich gerade von kulturpolitischer Seite evoziert. Siehe etwa die Rede des Kulturstaatsministers Bernd Neumann am 27. August 2006 anlässlich der Eröffnung der Ausstellung „Heiliges Römisches Reich Deutscher Nation 962 bis 1806“ im Deutschen Historischen Museum Berlin.

20 Milward, A. S.: The European Rescue of the Nation-State, Berkeley (Calif.), 1992. 
Mitte. Zugleich sind die großen Erzählungen von der europäischen Integration vom europäischen Phönix aus der Asche - alt geworden. Die neuen, ostmitteleuropäischen Mitgliedstaaten bringen eine ganz andere, häufig kontroverse Kriegsund Nachkriegserfahrung nach Europa mit hinein. Die westliche Erzählung der Integration kann nicht die ihrige sein. Die Erfahrung des und das Leiden am Kommunismus, der gewaltige Prosperitätsrückstand, aber auch die Spannungen, die das neu auszutarierende Verhältnis von Nationalstaat, Demokratie und europäischer Einigung auslöst, verändern die Bewegungsrichtung der Narration.

Andererseits beginnt das neue Europa zu Beginn des 21. Jahrhunderts seine Integration keineswegs voraussetzungslos, sondern es agiert in einem bereits objektivierten und institutionalisierten Rahmen, wie er durch eine mehr als fünfzigjährige Integrationsgeschichte bereits geschaffen worden ist. Ein gewisses $\mathrm{Ma}$ an Krisenfestigkeit darf man der EU daher durchaus zutrauen. Und was bisweilen allzu voreilig als das „Ende Europas" ${ }^{21}$ perhorresziert wird, dürfte im Kern eine Übergangskrise sein; eine Krise des Übergangs von den vertrauten Interessenstrukturen und Erzählkategorien des beendeten westeuropäischen Nachkriegsprojekts hin in die unbekannte Zukunft eines unübersichtlichen und pluralisierten, globalisierten und größeren Europas. ${ }^{22}$ Dessen ungeachtet bleibt natürlich offen, wie sich in der Zukunft die Dialektik zwischen den einzelnen europäischen Nationalstaaten und der supranational integrierten Ebene der EU vollziehen wird. Zwischen den einzelstaatlichen Stimmbürgerschaften und dem eher fiktiven Demos der Europäischen Union liegt jedenfalls nach wie vor eine breite Kluft, die auch die „Zukunftsfähigkeit“ der Gemeinschaft mit einem Fragezeichen versieht. ${ }^{23}$

\section{V.}

Die letzte Überlegung gilt denn auch der Frage, wieweit dieses Europa als Modell dienen kann für eine sich dynamisch entwickelnde „Weltgesellschaft “. In einem Punkt wird man sich dabei wahrscheinlich schnell einigen können: Als Modell besitzt Europa zweifellos viele Vorzüge - wie eingangs gesagt, wer woll-

21 Aus amerikanischer Sicht: Cohen-Tanugi, L.: The End of Europe?, in: Foreign Affairs 84,6 (2005), $55-$ 67.

22 Vgl. hierzu: Wirsching, A.: Europa als Wille und Vorstellung. Die Geschichte der europäischen Integration zwischen nationalem Interesse und großer Erzählung, in: Zeitschrift für Staats- und Europawissenschaften 4 (2006), 488-506.

23 Optimistisch hierzu Kuhnen, J. D.: Die Zukunft der Nationen in Europa. Ist das Zeitalter der Nationen und Nationalstaaten in Europa vorüber?, Berlin, 2009, v.a. 214-248. 
te heute nicht wie die Europäer leben? Unbestreitbar ist auch, dass das europäische Entwicklungsmodell die Idee des säkularen Staates in alle Welt exportiert hat - und damit seine auf Verrechtlichung, Rationalisierung und Bürokratisierung zielende Leittendenz.

Aber als Entwicklungsgeschichte taugt Europa wohl kaum zum Vorbild - oder welchem anderen Weltteil wollte man das Fegefeuer aus Nation, Ideologie und Gewalt andienen, das zum unauslöschlichen Kernbestand europäischer Erfahrung geworden ist? Diese Warnung vor einer gleichsam Europa-ähnlichen Entwicklung gilt umso mehr, als ja die einzelnen Bestandteile, die sie ermöglicht haben, im Grunde weltweit zur Verfügung stehen. Das gilt allemal für den Komplex von Nation, Staat und Grenze. Nationsbildungsprozesse, die einen sezessionistischen Sprengsatz in sich tragen, finden sich genügend außerhalb Europas. Ethnisch grundierte Territorialkonflikte brechen im Kaukasus und in Kaschmir, in Afrika und in Palästina auf. Immer wieder sind es ,,vorgestellte Gemeinschaften“, die sich der Realität entgegenstemmen und sie zu überwinden trachten. Seit dem Ende der europäischen Kolonialreiche und dem Zusammenbruch des Sowjetkommunismus haben sich die Bewegungsspielräume solcher Nationalismen gewaltig erweitert.

Gewaltig erweiterte sich seit 1989 jedoch auch der Radius der demokratischen Idee. Der enorme informationstechnologische und kommunikative Schub der letzten beiden Jahrzehnte hat die weltweite Rezeption des europäischen Modells weiter befördert. Und die globale Kommunikation verbreitet ein grundlegendes westlich-europäisches Prinzip, nämlich die Idee von der Gleichheit des Individuums. Niklas Luhmann hat diesen Gedanken zum Strukturmerkmal einer unaufhaltsamen Tendenz zur Weltgesellschaft erhoben. Denn wenn der aufklärerische Glaube an die Gleichheit der menschlichen Vernunft zutrifft, dann konstituiert Gleichheit auf Dauer auch die Gemeinschaft der Interessen. „Als Gleiche ... haben die Menschen gemeinsame Angelegenheiten. ${ }^{، 24}$ Wenn schließlich eine solche grundsätzliche Gleichheit anzunehmen ist, dann drängt das auf Gleichheit gegründete Modell aus sich selbst heraus auf weitere Ausdehnung. Die Idee der Gleichheit wird somit selbst zur Bedingung ihrer Universalisierung.

Tatsächlich will das auf den Ideen der Diesseitigkeit und der Gleichheit beruhende europäisch-westliche Modell selbst in der Weltgesellschaft politisch werden - vom Kongo bis nach Afghanistan. Indem es auf individuelle Menschen-

24 Luhmann, N.: Die Weltgesellschaft (1971), in: ders.: Soziologische Aufklärung, Bd 2: Aufsätze zur Theorie der Gesellschaft, 5. Aufl., Opladen, 2005, 63-88, hier 63. 
rechte und demokratische Verfassung rekurriert, wird sein rhetorischer Stil normativ und färbt sich moralisch. Seine Prämissen werden auf die Weltgesellschaft auch dann projiziert, wenn ein entsprechendes politisches System nicht hinreichend realisiert werden kann. Allerdings scheinen auch hier die Dinge im Fluss zu sein. Denn die Universalisierung des europäischen Gleichheitsgedankens vollzieht sich auf zwei Ebenen: zum einen individualistisch auf der Ebene der Menschenrechte; zum anderen kollektiv auf der Ebene der Staatenwelt.

Die Ausdifferenzierung eines Systems von prinzipiell gleichberechtigten Staaten hat in Europa seinen Anfang genommen und ist schon lange zum Organisationsmodell einer globalen internationalen Gemeinschaft geworden. Das gilt auch, wenn erstens längst nicht alle dieser Staaten demokratisch sind, und wenn man zweitens darüber streiten kann, inwieweit nicht das Subsystem Staat gegenüber anderen Subsystemen an Bedeutung verliert - Subsystemen, die im Übrigen ebenfalls europäischen Ursprungs sind. Häufig wird zum Beispiel die These vertreten, Staat und Politik träten gegenüber dem globalisierten weltwirtschaftlichen System zurück und büßten an Funktionen ein. Zwar lassen sich hierfür viele gute Argumente finden, aber möglicherweise bildet sich hier im globalen Maßstab ein Entwicklungsprozess ab, der aus der europäischen Geschichte durchaus bekannt ist. Auch in Europa stand die Politik längst nicht immer im Mittelpunkt der Gesellschaft, auch wenn dies der Selbstwahrnehmung ihrer Akteure vielleicht entsprechen mochte. Vielmehr differenzierten sich andere Subsysteme wie die Ökonomie dynamisch aus und entfalteten ihre systemische Eigenlogik. Gesellschaft erscheint damit als ein prekärer ,Zusammenhang eigenwilliger Funktionssysteme“ (Di Fabio). Und die neuere europäische Geschichte ist gekennzeichnet durch die immer wieder neu auszuhandelnde Balance zwischen politischem Regelungsanspruch und wirtschaftlicher Freiheit. Insofern ist auch das letzte Wort über die Rolle des Staatlich-Politischen in der globalisierten Weltwirtschaft noch nicht gesprochen. Und es wäre nicht das erste Mal in der Geschichte, dass einer voranschreitenden wirtschaftlichen Verflechtung die politische Integration nachfolgt. Die gegenwärtige Debatte über die Folgen der Finanz- und Wirtschaftskrise scheinen entsprechende Vermutungen zu bestätigen.

Zwar hat der Nationalstaat traditionell-europäischen Zuschnitts in der globalisierten Weltwirtschaft den Zenit seiner Steuerungsfunktionen wohl überschritten. Aber die spezifische Antwort, die Europa auf das Erfordernis eines postnationalstaatlichen Ordnungsmodells gefunden hat - die Integration -, könnte auch im globalen Maßstab eine Wirkung entfalten. Dafür spricht nicht nur das fortbestehende Ausdehnungspotential der Europäischen Union selbst, das sich aus offen- 
kundig ungebrochener Attraktivität speist und über das alte Kerneuropa bereits weit hinausweist. Hinzu kommt die wachsende Rolle intergouvernementaler Körperschaften, aber auch der NGOs, deren Zahl sich nicht zufällig seit dem Ende des Kommunismus deutlich erhöht hat. Gab es zum Beispiel im Jahre 1987 „nur“ 4.235 NGOs, so zählte man im Jahre 2004 bereits 7.306 und im Jahre 2007 7.628 Nichtregierungsorganisationen. ${ }^{25}$ Sie agieren weltweit und fehlen in keinem Diskurs über global governance.

Auch wenn keineswegs immer ganz klar ist, welche Funktionen internationale, intergouvernementale und supranationale Körperschaften sowie NGOs übernehmen, so können sie doch als Zeichen einer wachsenden globalen Verflechtung gewertet werden. In dem Maße, in dem sie allgemeine und universelle Gesellschaftsinteressen vertreten, werden sie zum Symptom einer sich entfaltenden und sich kommunikativ verdichtenden Weltgesellschaft. Zwar ist eine Weltgesellschaft in diesem Sinne, die das Prinzip der Gleichheit durch globale und immer intensivere Kommunikation verbreitet, durchaus etwas Fiktives. Aber eben der Eindruck, es handle sich um etwas, das erst noch zu erreichen wäre und doch nicht erreicht werden kann, trägt zur stetigen weiteren Universalisierung bei.

Werden also Nation, Demokratie und Menschenrechte im Rahmen globaler Integration zusammenkommen und eine gewissermaßen Europa-ähnliche Friedensordnung hervorbringen?

Einiges spricht dafür: Alle Staaten der Vereinten Nationen verstehen sich in irgendeiner Form als Nationalstaaten, auch wenn es sich faktisch um Vielvölkerstaaten handelt oder sie in ihren Grenzen starke ethnische Minderheiten haben; und ihre Regierungen berufen sich fast alle irgendwie auf das Volk als die Quelle ihrer Legitimität. In Zeiten der globalen Kommunikation registriert die zusammengerückte Weltöffentlichkeit die Verletzung von Menschenrechten und Menschenwürde mit unmittelbarer und intensiver Aufmerksamkeit. All dies übt eine Art wachsenden Disziplinierungsdruck aus - zumindest auf der semantischen Ebene. Wer gegen die Norm verstößt, muss sich gesondert legitimieren.

Spätestens hier stellt sich freilich die Frage, ob es sich überhaupt um einen wünschenswerten Vorgang handeln kann. Ist nicht die europäische Rhetorik von Demokratie und Menschenrechten die neue Form eines alten Bekannten: des rücksichtslosen Eurozentrismus, der die historische Legitimität anderer Kulturen nicht anerkennen und Vielfalt nicht zulassen will? Sollten wir nicht vielmehr auf 
die „multiple modernities“ differenter Kulturen blicken als auf die Durchsetzungskräfte eines europäischen Entwicklungsmodells?

Beides muss sich freilich nicht ausschließen: Weder kann sich ein Entwicklungsmodell unilateral einfach durchsetzen, noch erweisen sich andere Kulturen als undurchlässig gegenüber anderen. Wer zum Beispiel behaupten würde, China oder die islamische Welt seien aus Gründen ihrer Kulturgrenzen definitiv nicht in der Lage, eine grundrechtbasierte Zivilgesellschaft zu entwickeln, würde ein höchst problematisches, allzu eindimensionales, ja statisches Bild zeichnen; und die schiere Dynamik der Globalisierung lehrt uns eines besseren. Die außereuropäischen Regionen waren und sind aufnahmefähig für europäisch-westliche Einflüsse. Umgekehrt bleibt Europa dauerhaft herausgefordert, im Lichte globaler Erfahrungen die Prämissen und Folgen seiner Entwicklung zu reflektieren und auf den Prüfstand zu stellen.

Die Folge ist jene kontinuierliche Interaktion, die sich in der globalisierten Welt unaufhaltsam beschleunigt und auf deren Basis sich neue Konstellationen und neue Institutionen herausbilden. Die Ergebnisse bleiben allerdings unsicher. Auf der einen Seite hat das europäische Modell seit den 1980er Jahren im Kontext globaler Kommunikation an Attraktivität und an Ausdehnungskraft gewonnen. Auf der anderen Seite bestehen die Schattenseiten fort. Die ,dunkle Seite der Demokratie“, die dem Ethno-Nationalismus entspringt, bricht weltweit immer wieder hervor und hat Europa in Jugoslawien selbst noch einmal heimgesucht. Auch kann man fragen, ob das europäische Modell nicht nach wie vor dazu tendiert, im Zuge eines zügellosen, auf Rationalisierung und Bürokratie zielenden Effizienzdenkens das „stahlharte Gehäuse“, als das Max Weber die Moderne bezeichnete, immer weiter zu reproduzieren. Und ob eine globale Ausdehnung dieses Gehäuses wünschenswert ist, erscheint mehr als fraglich. Insofern wird sich die Geschichte einer Weltgesellschaft, in der Europa seine Rolle spielt, nicht weniger ambivalent vollziehen können als die Geschichte des europäischen Entwicklungsmodells selbst. 